\title{
MicroRNA-137 suppresses tumor growth in epithelial ovarian cancer in vitro and in vivo
}

\author{
LIYU ZHANG $^{1}$, ZHIHONG LI $^{1}$, FENGCHUN GAI ${ }^{2}$ and YANPING WANG ${ }^{1}$ \\ Departments of ${ }^{1}$ Obstetrics and Gynecology and ${ }^{2}$ Infectious Diseases, The Affiliated Hospital, \\ Changchun University of Chinese Medicine, Changchun, Jilin 130117, P.R. China
}

Received July 21, 2014; Accepted April 8, 2015

DOI: $10.3892 / \mathrm{mmr} .2015 .3756$

\begin{abstract}
Epithelial ovarian cancer (EOC) remains a major gynecological problem, with a poor 5-year-survival rate due to distant metastases. The identification of microRNAs (miRNAs) may provide a novel avenue for diagnostic and treatment regimens for EOC. Several miRNAs have been reported to be involved in the progression of EOC, among which miRNA (miR)-137 has been observed to be downregulated in the ovarian tissues of patients with EOC. However, the functions of miR-137 in EOC cell apoptosis, migration and invasion remain to be elucidated. In the present study, the expression of miR-137 was measured in clinical ovarian cancer specimens and cell lines using reverse transcription-quantitative polymerase chain reaction. The role of miR-137 in the growth and survival of the SKOV3 human ovarian cancer cell line was determined using several in vitro approaches and in nude mouse models. The results demonstrated that the expression of miR-137 was downregulated in the ovarian cancer specimens and cell lines. It was also observed that enforced expression of miR-137 in the EOC cell lines decreased cell proliferation, clonogenicity, migration and invasion, and induced G1 arrest and cell apoptosis in vitro. Notably, the enforced expression of miR-137 suppressed tumor growth in the nude mice models. These findings suggested that miR-137 may act as a tumor suppressor and be used as a potential therapeutic agent for the treatment of EOC.
\end{abstract}

\section{Introduction}

Human ovarian cancer is the fourth most common type of cancer among females worldwide, and $\sim 90 \%$ of cases of ovarian cancer are epithelial ovarian cancer (EOC) $(1,2)$. Despite the

Correspondence to: Professor Yanping Wang, Department of Obstetrics and Gynecology, The Affiliated Hospital, Changchun University of Chinese Medicine, 1035 Boshuo Road, Changchun, Jilin 130117, P.R. China

E-mail: wyanping721@163.com

Key words: epithelial ovarian cancer, microRNAs, microRNA-137, tumor growth increases use of surgery and chemotherapy to treat EOC, the outcome remains poor, with a 30-40\% 5-year-survival rate in patients with EOC, due to the lack of specific symptoms in the early stages of the disease, and a lack of effective tumor biomarkers and effective treatment strategies $(3,4)$. Therefore, identifying the molecular mechanisms of EOC carcinogenesis may improve current understanding of the pathogenesis of EOC and, ultimately, identify novel diagnostic and/or prognostic markers.

MicroRNAs (miRs/miRNAs) are small (19-25 nt), endogenous non-coding RNAs, and are novel post-transcriptional regulators of gene expression through binding to the 3'-untranslated region of target genes via their own RNA-induced silencing complexes (5-8). The actions of miRNAs are varied, as they have been observed to be involved in a multitude of physiological processes, including the cell cycle, metabolism, angiogenesis, differentiation, apoptosis and proliferation (9-14). Additionally, increasing reports have indicated that miRNAs are involved in tumorigenesis and cancer metastasis by regulating the expression of their target oncogenes or tumor suppressor genes $(15,16)$. For example, miR-135a acts as a potential tumor suppressor inhibiting EOC cell proliferation, migration and invasion by decreasing the expression of the Homeobox A10 oncogene (17), and miR-124 markedly inhibits the motility of ovarian cancer cells in vitro by downregulating the protein expression of the sphingosine kinase 1 oncogene (18).

An increasing number of reports have indicated that a number miRNAs are involved in several aspects of the pathogenesis of EOC, including cell cycle control, invasion, migration, resistance to chemotherapy and radiotherapy, and cell apoptosis. It has been reported that the miR-15a, miR-16 (19), miR-221/222 (20) and miR-200 families (7) are upregulated, while miRNA-34 (21), miR-124 (18), miR-145 (22) and miR-137 families (23) are downregulated in EOC. miR-137 is located on chromosome 1p21.3 and lies across a large $\mathrm{CpG}$ island, which is associated with certain diseases and has been found to be abnormally expressed in lung cancer (24), oral squamous cell carcinoma (25), gastric cancer cell lines (26), colorectal cancer (27), neuroblastoma (28) and breast cancer (29). At present, only one study has reported that miR-137 is differentially expressed between normal ovarian and ovarian cancer tissues through quantitative polymerase chain reaction (qPCR), which also found 
that miR-137 negatively regulates the astrocyte elevated gene-1 (AEG-1) oncogene and suppresses ovarian cancer cell proliferation and clonogenicity (23). However, the function of miR-137 in the apoptosis, migration and invasion of EOC cells remains to be elucidated Validation of the function of miR-137 in ovarian cancer, particularly in EOC is required, therefore, the aim of the present study was to characterize the expression of miR-137, identify its detailed function in EOC cells in vitro and in vivo to determine its utility in EOC diagnosis and therapy.

\section{Patients and methods}

Patients and tissue samples. The procedures used in the present study were approved by the Ethical Committee of the Affiliated Hospital, Changchun University of Chinese Medicine (Changchun, China). All patients were informed and agreed to be involved in the present study. All clinical investigations were performed according to the principles detailed in the Declaration of Helsinki.

Malignant ovarian tumor tissues and their adjacent non-tumorous samples were obtained from 30 consecutive patients with EOC, who underwent curative ovarian resection between June 2012 and March 2014 at the Affiliated Hospital, Changchun University of Chinese Medicine. Patients with a previous or secondary malignancy, or who had previously undergone chemotherapy or radiotherapy treatment were excluded from the investigation. Clinicopathological information was collected from the clinical data of patients diagnosed with OEC (as listed in Table I). The tumor tissues were staged according to the tumor, node, metastasis staging system of the Union for International Cancer Control (30).

Cell lines and cell culture. The SKOV3 human ovarian cancer cell line, with a high metastatic potential, (31) was obtained from the Shanghai Institute of Cell Biology, China Academy of Sciences (Shanghai, China). The HEY human ovarian cancer cell line and normal human ovarian surface epithelial (OSE) cells were obtained from American Type Culture Collection (Manassas, VA, USA). The SKOV3 and HEY cells were cultured in RPMI-1640 medium (Sigma-Aldrich, St. Louis, MO, USA), supplemented with $10 \%$ fetal bovine serum (FBS; Gibco Life Technologies, Carlsbad, CA, USA) in a humidified atmosphere of $5 \% \mathrm{CO}_{2}$ at $37^{\circ} \mathrm{C}$. The OSE cells were cultured in Dulbecco's modified Eagle's medium-F12 growth medium (Sigma-Aldrich) with 15\% FBS and 5\% penicillin-streptomycin (Sigma-Aldrich). The complete media was generally replaced every 2-3 days.

miRNA transfection. The miR-137 mimic and negative control miRNA (mirVanaR miRNA mimic/inhibitor; Life Technologies, Grand Island, NY, USA) were transiently transfected into the SKVO3 ovarian cell lines in 6-well plates using Oligofectamine ${ }^{\mathrm{TM}}$ transfection reagent (Invitrogen Life Technologies, Carlsbad, CA, USA), according to the manufacturer's instructions, at a concentration of $100 \mathrm{nM}$.

Reverse transcription (RT)-qPCR. The isolation of total RNA from the cells and ovarian tissues was performed using QIAzol lysis reagent and an miRNeasy mini kit (Qiagen,
Valencia, CA, USA), according to the manufacturer's instructions. The RNA was reverse-transcribed using a One Step Prime script miRNA cDNA synthesis kit (Qiagen) and then quantified using RT-qPCR with SYBR Premix Ex Taq (Takara Bio Inc., Dalian, China). All the PCR reactions were detected using the ABI 7900 Fast system (Applied Biosystems, Foster City, CA, USA). The primers used in the RT-qPCR reaction were as follows: miR-137, sense 5'-GCGCTTATTGCTTAA GAATAC-3' and antisense 5'-CAGTGCAGGGTCCGA GGT-3'; and U6, sense 5'-CTCGCTTCGGCAGCACATATA CT-3' and antisense 5'-ACGCTTCACGAATTTGCGTGTC-3'. Amplification of miR-137 and U6 was performed with 1 cycle at $95^{\circ} \mathrm{C}$ for $5 \mathrm{~min}$ and 40 cycles of $95^{\circ} \mathrm{C}$ for $15 \mathrm{sec}$ then $55^{\circ} \mathrm{C}$ for $60 \mathrm{sec}$. The expression levels of U6 were determined as an internal control. The PCR efficiencies were calculated using a relative standard curve, derived from a complementary DNA mixture, and provided regression coefficients of $>0.95$. The relative quantification of each miRNA was presented as the fold change following normalization to the U6 RNA, with the $2^{-\Delta \Delta \mathrm{Ct}}$ equation using Rotor-Gene 6000 series software 1.7 (Qiagen). All experiments were performed in triplicate to reduce curve-derived variance.

Cell proliferation and colony formation assays. To measure the effect of miR-137 on cell proliferation, a Cell Counting kit-8 (CCK-8) assay was performed (Dojindo Laboratories, Kumamoto, Japan). Briefly, the SKOV3 cells were seeded into 24 -well plates at a density of $5 \times 10^{3}$ cells/well. The SKOV3 cells were them incubated in $10 \%$ CCK8 and diluted in normal culture medium at $37^{\circ} \mathrm{C}$ until visual color conversion occurred. The absorbance in each well was measured on a Wellscan MK3 microplate reader set at $450 \mathrm{~nm} \mathrm{24,48,} 72$ and $96 \mathrm{~h}$ after transfection using an enzyme-linked immunosorbent assay (ELISA) reader (Thermo Labsystems, Helsinki, Finland). The experiment was performed at least three times with similar results.

For the colony formation assay, the cells were seeded in 6 -well plates at a low density $\left(1 \times 10^{3}\right.$ cells/well) and cultured for 7 days. Subsequently, the cells were fixed with $4 \%$ paraformaldehyde for $20 \mathrm{~min}$ and were counted following staining with $1 \%$ crystal violet (Sigma-Aldrich). The experiments were performed in triplicate wells at least three times.

Cell cycle and cell apoptosis assay. For analysis of the cell cycle, the SKOV3 cells were transfected with either miR-137 or negative control miRNA. At $48 \mathrm{~h}$ post-transfection, the cells were collected by trypsinisation, washed with ice-cold phosphate-buffered saline (PBS; pH 7.2; Sigma-Aldrich), and the fixed cells were then incubated with the DNA binding dye propidium iodide (20 $\mu \mathrm{g} / \mathrm{ml}$; Sigma-Aldrich) and RNase $(1.0 \mathrm{mg} / \mathrm{ml})$ for $30 \mathrm{~min}$ at $37^{\circ} \mathrm{C}$ in the dark, followed by analysis using flow cytometry (FACSCalibur; BD Biosciences, Mansfield, MA, USA). The experiments were performed in triplicate.

For analysis of apoptosis, the SKOV3 cells were collected and diluted to a concentration of $1 \times 10^{6}$ cells $/ \mathrm{ml}$, then washed three times with ice cold PBS $48 \mathrm{~h}$ post-transfection. The cells were incubated with phycoerythrin (PE)-Annexin-V and 7AAD using the PE Annexin-V apoptosis detection kit I (BD Pharmingen, CA, USA), according to the manufacturer's 
Table I. Correlation between the relative levels of miR-137 in ovarian epithelial tissues and clinicopathological features of patients with ovarian epithelial cancer.

\begin{tabular}{|c|c|c|c|}
\hline Feature & Number of patients & Relative level of miR-137 (2- $\left.2^{-\Delta \Delta \mathrm{Ct}}\right)$ & P-value \\
\hline \multicolumn{4}{|c|}{ Age (years) } \\
\hline$<55$ & 18 & $4.586 \pm 0.435$ & \multirow[t]{2}{*}{ NS } \\
\hline$\geq 55$ & 12 & $4.389 \pm 0.427$ & \\
\hline \multicolumn{4}{|c|}{ Regional lymph nodes } \\
\hline Yes & 13 & $3.046 \pm 0.286$ & \multirow[t]{2}{*}{$\mathrm{P}<0.01$} \\
\hline No & 17 & $4.912 \pm 0.792$ & \\
\hline \multicolumn{4}{|c|}{ TNM clinical stage } \\
\hline I-II & 19 & $5.035 \pm 0.418$ & \multirow[t]{2}{*}{$\mathrm{P}<0.01$} \\
\hline III-IV & 11 & $3.382 \pm 0.288$ & \\
\hline
\end{tabular}

miR, microRNA; TNM, tumor, node, metastases cancer staging, according to the Union for International Cancer Control System; NS, no significance ( $\mathrm{P} \geq 0.05 ;<55$-years, vs. $\geq 55$-years $)$.

instructions, and then analyzed using fluorescence-activated cell sorting (FACS). The experiments were performed in triplicate. In addition, caspase-3 activity was detected using an ELISA, as an additional indicator of apoptosis.

Measurement of caspase-3 activity. The SKOV-3 cells were plated in 6-well plates and transfected with the miR-137 mimics or corresponding negative control. At $48 \mathrm{~h}$ post-incubation, the cells were harvested and lysed using lysis buffer (Beyotime Institute of Biotechnology, Haimen, China) on ice for $20 \mathrm{~min}$. The cell lysates were centrifuged at 20,000 x g for $15 \mathrm{~min}$ at $4^{\circ} \mathrm{C}$. The protein concentration was measured using a Bradford protein assay kit (Beyotime Institute of Biotechnology). The activation of caspase-3 activity in the SKOV-3 cells was determined using a caspase-3 activity assay kit (Beyotime Institute of Biotechnology), according to the manufacturer's instructions. The experiment was performed at least three times in triplicate.

Wound-healing assays. The SKOV3 cells were grown to $80-90 \%$ confluence in 24-well plates and treated with miR-137 mimics or the corresponding negative control. At $24 \mathrm{~h}$ post-transfection, linear scratch wounds were created on the confluent cell monolayers using a $200 \mu \mathrm{l}$ pipette tip. To prevent the cells from entering the cell cycle prior to wounding, the cells were maintained in serum-free RPMI-1640 medium. To visualize the migrating cells and wound healing, images were captured at 0 and 24 h. For visualization, five field areas were randomly selected from each well, and three wells of each cell group were analyzed.

Cell invasion assay. For the invasion assay, Transwell migration chambers ( $8-\mu \mathrm{m}$ pore filter; BD Biosciences) were coated with Matrigel (BD Biosciences) and incubated at $37^{\circ} \mathrm{C}$ for $4 \mathrm{~h}$, allowing it to solidify. At $24 \mathrm{~h}$ post-transfection with the miR-137 mimics, 5x10 5 SKOV3 cells, suspended in serum-free RPMI-1640 medium, were added to the upper chamber and medium, containing $10 \%$ FBS, was added to the lower chamber as a chemoattractant. After $48 \mathrm{~h}$, the non-invading cells were gently removed using a cotton swab, and the invasive cells located on the lower surface of the chamber were stained with $0.1 \%$ crystal violet in $20 \%$ methanol (Sigma-Aldrich). The level of invasiveness was determined by counting the number of penetrating cells under a Nikon phase-contrast microscope (Nikon TMS; Nikon, Tokyo, Japan) and counted in $>10$ fields of view (magnification, $\mathrm{x} 200$ ). The invasion of the cells without any treatment was determined as $100 \%$.

Western blot analysis. For the western blot analyses, $48 \mathrm{~h}$ post-transfection, the cells were harvested and lysed via incubation on ice for $30 \mathrm{~min}$ in lysis buffer, containing $25 \mathrm{mM}$ Tris- $\mathrm{HCl}$ (pH 8.0), 1\% Nonidet $\mathrm{P} 40,0.5 \%$ sodium deoxycholate, $0.1 \%$ sodium dodecylsulfate (SDS) and $125 \mathrm{mM}$ $\mathrm{NaCl}$, containing complete protease inhibitor cocktail (Roche, Mannheim, Germany). The protein concentration was determined using a bicinchoninic acid protein assay kit (KeyGEN Biotech, Nanjing, China) using a c-globulin standard curve. Equal quantities of protein $(20 \mu \mathrm{g} /$ lane $)$ from the cell lysates were separated on $10 \%$ SDS-polyacrylamide gels and transferred onto nitrocellulose membranes (Santa Cruz Biotechnology, Inc., Santa Cruz, CA, USA). The membrane was incubated for $2 \mathrm{~h}$ in PBS with $0.1 \%$ Tween-20 and 5\% nonfat skim milk to block nonspecific binding. Subsequently, the membranes were incubated overnight at $4^{\circ} \mathrm{C}$ with the following antibodies: Mouse polyclonal anti-human matrix metalloproteinase (MMP)-2 (1:1,000; cat. no. 4022s; Cell Signaling Technology, Inc., Danvers, MA, USA) and mouse monoclonal anti-human MMP-9 (1:2,000; cat. no. sc-12759; Santa Cruz Biotechnology, Inc.). Mouse monoclonal anti-human GAPDH (1:10,000; cat. no. sc-365062; Santa Cruz Biotechnology, Inc.) was used as a loading control. The membranes were then incubated with polyclonal goat anti-mouse horseradish peroxidase-conjugated immunoglobulin G (1:10,000; cat. no. sc-8454; Santa Cruz Biotechnology, Inc.) for $2 \mathrm{~h}$ at room temperature and the proteins were detected using an enhanced chemiluminescence detection kit (Thermo Fisher Scientific, Rockford, IL, USA) and then visualized using a Molecular Imager ${ }^{\circledR}$ ChemiDoc $^{\mathrm{TM}}$ $\mathrm{XRS}+$ system (Bio-Rad Laboratories, Inc., Hercules, CA, USA). 
Tumor growth in vivo. A total of 30 male BALB/c mice (5-6-weeks old; 18-20 g), were purchased from HFK Bio-Technology, Co., Ltd. (Beijing, China) and were maintained under specific pathogen-free conditions with ad libitum access to food and water at room temperature with $45-70 \%$ humidity. Subsequently, $2 \times 10^{6}$ of the SKOV3 cells stably overexpressing miR-137 or the corresponding negative control cells were suspended in $50 \mu \mathrm{l}$ PBS and injected into the flanks of the mice $(\mathrm{n}=10)$. The mice were monitored weekly for tumor growth. Tumor size was measured every week, and tumor volume was calculated as $0.5236 \mathrm{x}$ width $^{2} \mathrm{x}$ length. At 4 weeks post-inoculation, the mice were sacrificed via cervical dislocation. The tumors were resected and weighed, and a section of each tumor was used to measure the levels of miR-137 using RT-qPCR. All animal experiments were performed, according to the standards of animal care as outlined in the Guide for the Care and Use of Changchun University of Chinese Medicine, and were approved by the Ethics Committees of the Affiliated Hospital, Changchun University of Chinese Medicine.

Statistical analysis. The data are expressed as the mean \pm standard deviation of at least three independent experiments. Differences between groups were compared using one-way analysis of variance or a two-tailed Student's t-test with SPSS version 16.0 (SPSS, Inc., Chicago, IL, USA) and GraphPad Prism version 5.01 (GraphPad, San Diego, CA, USA) software for Windows ${ }^{\circledast}$. $\mathrm{P}<0.05$ was considered to indicate a statistically significant difference.

\section{Results}

Expression of miR-137 is reduced in highly metastatic ovarian cancer cell lines and clinical tumor tissues. To determine the expression levels of miR-137 in the progression of ovarian cancer, the expression levels between malignant ovarian tumor tissues and adjacent non-tumorous tissues were compared using RT-qPCR. As shown in Fig. 1A, the results revealed that the expression of miR-137 was decreased in the ovarian cancer samples $(\mathrm{P}<0.01)$, compared with the non-tumorous tissue. Furthermore, the correlation between the expression of miR-137 and various clinicopathological factors, including patient age, presence of lymph node metastasis and clinical stage, was examined (Table I). Patients with lymph node metastases were found to exhibit significantly lower expression levels of miR-137, compared with those without metastases $(\mathrm{P}<0.01)$. The expression levels of miR-137 were also found to be lower in patients with clinical stage III-IV carcinoma, compared with stage I-II $(\mathrm{P}<0.01)$. No correlation was identified between the expression of miR-137 and patient age.

The levels of miR-137 were further detected in two ovarian cancer cell lines (SKOV3 and HEY) and normal human OSE cells using RT-qPCR. Compared with the OSE cells, the levels of miR-137 were significantly reduced, to different extents, in the SKOV3 cells and HEY cells (Fig. 1B), with markedly lower levels in the SKOV3 cells with a high metastatic potential, in particular. These results suggested that the expression of miR-137 was significantly decreased in the human ovarian cancer specimens and cell lines, and may be involved in EOC metastasis.
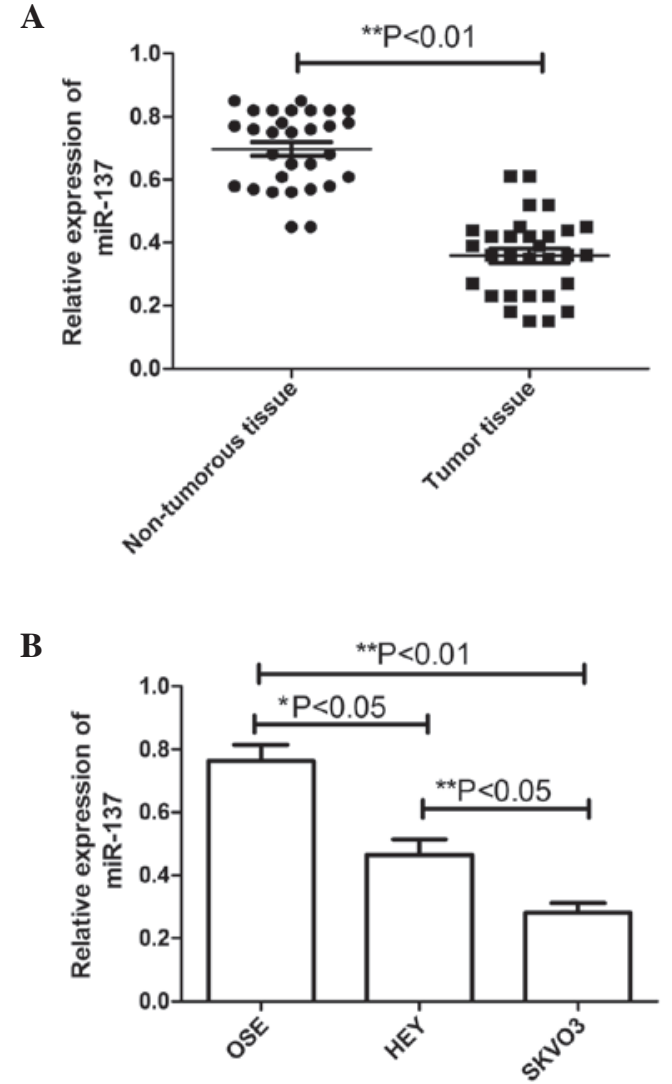

Figure 1. Expression of miR-137 in cell lines and tissues in ovarian cancer. (A) Expression of miR-137 in ovarian tumor tissues and adjacent non-tumorous ovarian tissues. (B) Relative expression of miR-137 in SKOV3 and HEY ovarian cancer cell lines and normal OSE cells. Data are presented as the mean \pm standard deviation of three individual experiments. ${ }^{*} \mathrm{P}<0.05$ and ${ }^{* *} \mathrm{P}<0.01$. miR, microRNA; OSE cells, ovarian surface epithelial cell.

miR-137 inhibits EOC cell proliferation and colony formation. In view of the reduced expression of miR-137 in SKOV3 cells, the effect of miR-137 on the proliferation of SKOV3 cells was examined. The miR-137 mimic and corresponding negative control were transfected into the SKOV3 cells, following which CCK-8 assays were performed. As shown in Fig. 2A, the viability of the SKOV3 cells was markedly decreased following transfection with the miR-137 mimic $(\mathrm{P}<0.05)$, compared with the negative control. The effect of the miR-137 mimic on cell proliferation was observed from day 2, becoming more marked on days 3 and 4 ( $\mathrm{P}<0.01$; Fig. 2A).

In addition, the present study determined the effect of miR-137 on cell colony formation, which demonstrated that the cells transfected with the miR-137 mimics exhibited significantly inhibited cell colony formation, compared with cells transfected with the negative control $(\mathrm{P}<0.01$; Fig. $2 \mathrm{~B})$. These findings suggested that overexpression of miR-137 markedly inhibited cell proliferation and colony formation in the SKOV3 cells.

miR-137 induces G1 arrest and cell apoptosis. In order to determine the effects of miR-137 on the cell cycle, FACScan flow cytometry assays were performed. The results revealed that the G1-phase cell population was increased in the cells transfected with the miR-137 mimics, compared with the cells transfected with the negative control $(\mathrm{P}<0.05$; Fig. $3 \mathrm{~A}$ and $\mathrm{B})$. 

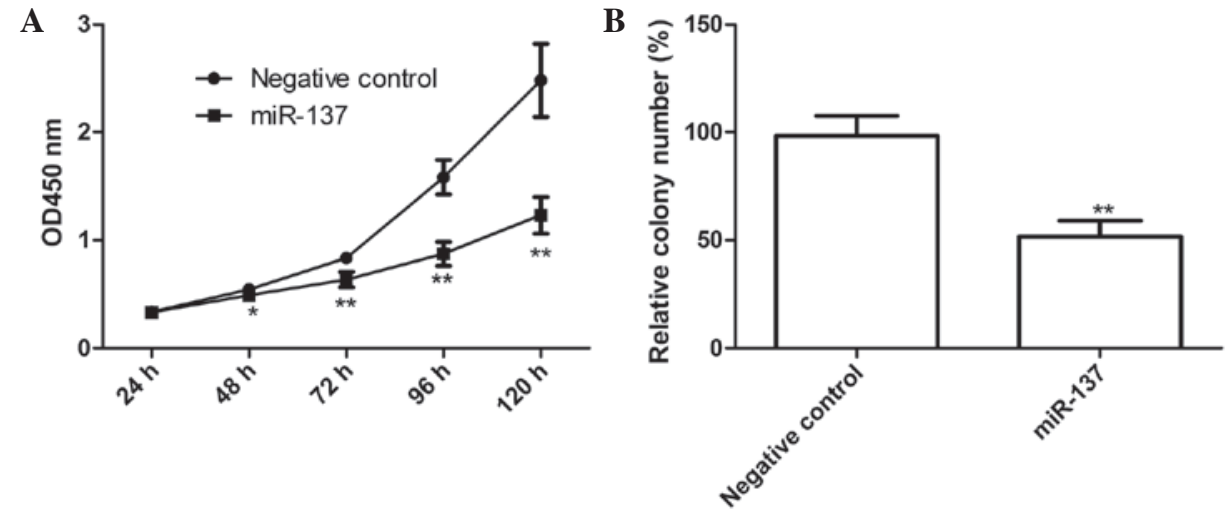

Figure 2. miR-137 inhibits cell proliferation and cell colony formation in SKOV3 cells. (A) A cell counting kit-8 assay was performed to analyze the effect of miR-137 on the cell proliferation of SKOV3 cells. (B) Effects of miR-137 on cell colony formation were determined. Data were from at least three independent experiments. ${ }^{*} \mathrm{P}<0.05$ and ${ }^{* *} \mathrm{P}<0.01$, vs. negative control. miR, microRNA; OD, optical density.

A

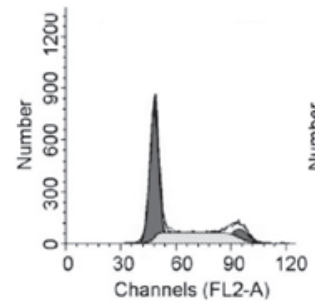

Negative control

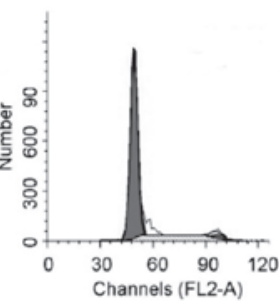

$\operatorname{miR}-137$

C

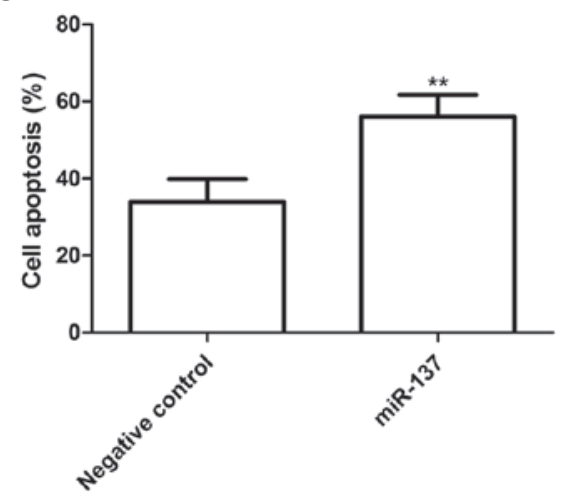

B

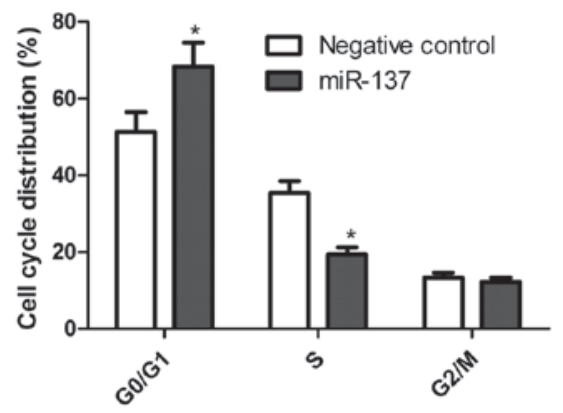

D

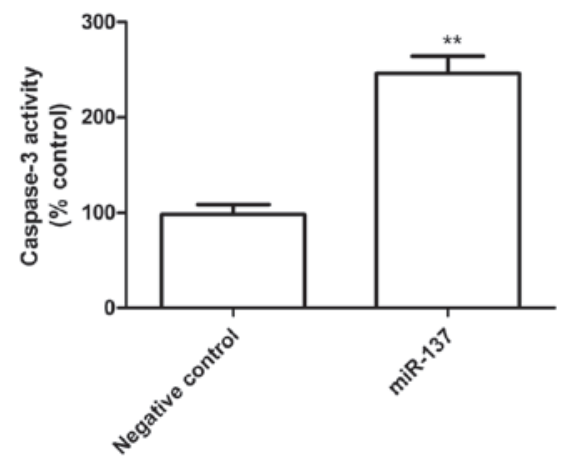

Figure 3. miR-137 induces changes in cell cycle distribution and cell apoptosis in SKOV3 cells. (A) Cell DNA content distribution in each phase. (B) Percentages of cells distributed in each phase of the cell cycle. (C) Cell apoptosis was determined following transfection of the cells with miR-145 or the corresponding negative control. (D) Caspase-3 activity was determined using an enzyme linked immunosorbent assay. " $\mathrm{P}<0.05$ and ${ }^{* *} \mathrm{P}<0.01$, vs. negative control. miR, microRNA.

The role of miR-137 in SKOV3 cell apoptosis was also assessed, which revealed that apoptosis was significantly induced in the SKOV3 cells transfected with the miR-137 mimics, compared with cells transfected with the negative control $(\mathrm{P}<0.01$; Fig. 3C). Finally, the effects of miR-137 on caspase-3 activity were analyzed using an ELISA. As shown in Fig. 3D, caspase-3 activity in the cells transfected with the miR-137 mimics was significantly increased compared with those transfected with the negative control $(\mathrm{P}<0.01)$.

miR-137 inhibits cell migration and invasion in SKOV3 cells. To ascertain the inhibitory effect of miR-137 on cell motility in vitro, a wound-healing assay was performed. A scratch was introduced into confluent monolayers of SKOV3 cells transfected with the miR-137 mimic or the corresponding negative control, and the time-dependent movement of the cells into the injured area was monitored microscopically. After $24 \mathrm{~h}$, the SKOV3 cells transfected with the miR-137 mimics exhibited significantly decreased migration, compared with the cells transfected with the negative control $(\mathrm{P}<0.01$; Fig. 4A).

The ability of miR-137 to reduce the invasiveness of SKOV3 cells was further investigated using a Transwell system assay. The results demonstrated that overexpression of miR-137 significantly inhibited cell invasion ( $\mathrm{P}<0.01$; Fig. 4B). 
A

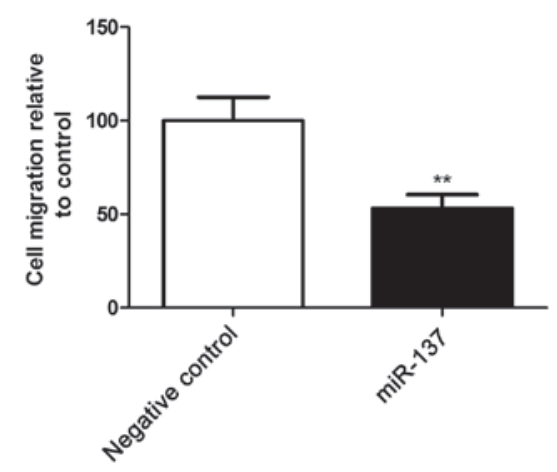

C

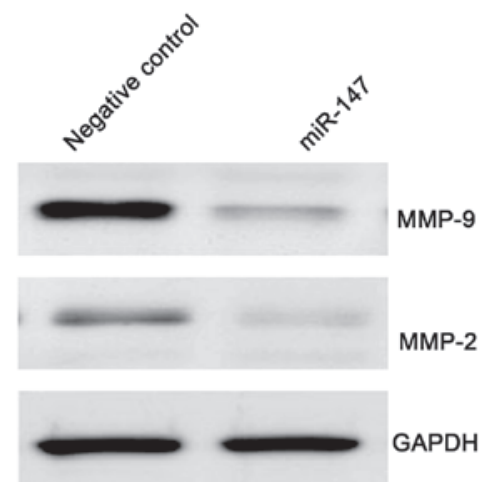

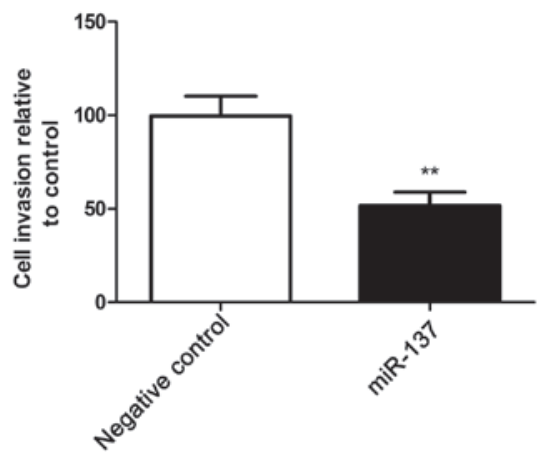

D

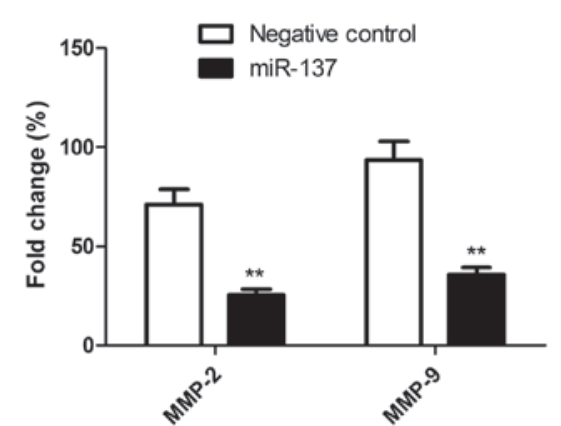

Figure 4. miR-137 inhibits cell migration and invasion in SKOV3 cells. (A) Cell migration was determined using a wound-healing assay. (B) Cell invasion was determined using a Matrigel Transwell assay. (C) Western blot analysis of the protein expression levels of MMP-2 and MMP-9 following transfection with miR-137 or the corresponding negative control. GAPDH was used as an internal control. (D) Relative quantification of the protein levels of MMP-2 and MMP-9 by densitometric analysis, ${ }^{*} \mathrm{P}<0.05$ and $^{* *} \mathrm{P}<0.01$, vs. negative control. miR, microRNA; MMP, matrix metalloproteinase.

A

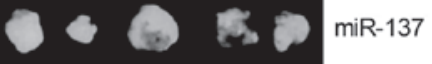 C) Fis 20 Negative control}

C

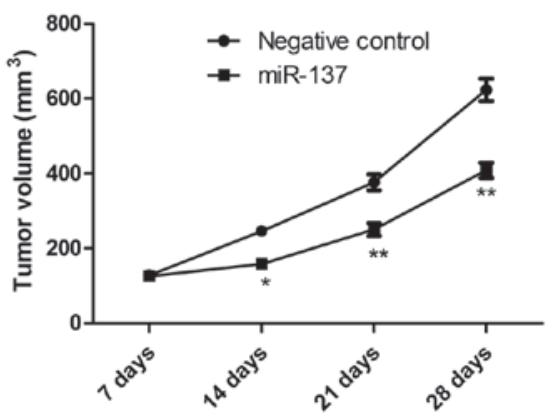

B

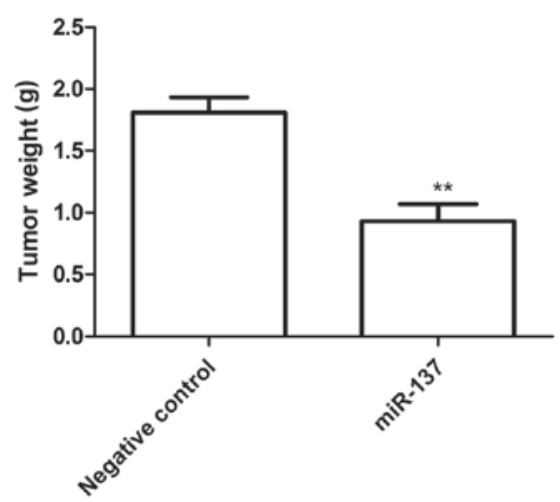

D

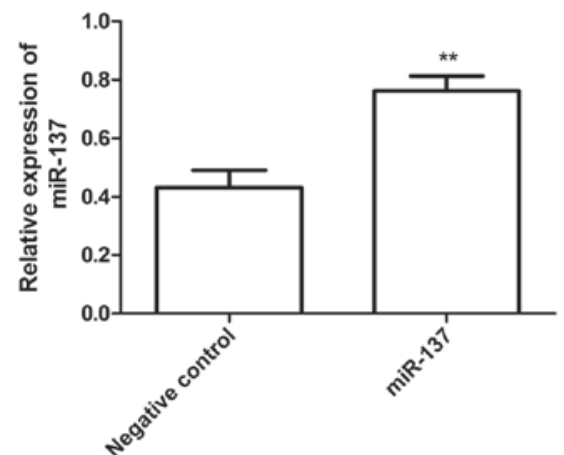

Figure 5. miR-137 suppresses tumor growth in nude mice. (A) miR-137-overexpressing or negative control SKOV3 cells were injected subcutaneously into nude mice $(n=10)$. The mice were sacrificed and the tumors were recovered 28 days after implantation. Representative graphs of tumor volumes are shown. (B) Tumor wet weights were measured following sacrifice at day 28. (C) Tumor growth cures over the 28-day period were established, based on the tumor sizes measured each week. (D) Reverse transcription-quantitative polymerase chain reaction analyses for miR-137 in the tumor tissues. ${ }^{*} \mathrm{P}<0.05$ and ${ }^{* *} \mathrm{P}<0.01$, vs. negative control. miR, microRNA. 
Furthermore, the effects of miR-137 on the expression levels of the cell invasion relevant proteins, MMP-2 and MMP-9, were analyzed using western blot analysis As shown in Fig. 4C and 4D, compared with the cells transfected with the negative control, the protein expression levels of MMP-2 and MMP-9 were significantly decreased in the cells transfected with the miR-137 mimics $(\mathrm{P}<0.05)$.

miR-137 suppresses tumor growth in a nude mouse model. To further examine the association between miR-137 and tumorigenesis in vivo, SKOV3 cells stably overexpressing miR-137 or negative control were injected subcutaneously into nude mice $(\mathrm{n}=10)$. The tumor volume was measured once a week, and mice were sacrificed 28 days after tumor cell implantation. As shown in Fig. 5A, the miR-137-overexpressing tumors were significantly smaller than tumors in the negative control group. The average volume and weight of the miR-137-overexpressing tumors were significantly reduced, compared with the negative control group $(\mathrm{P}<0.05$; Fig. 5B and 5C). The level of miR-137 in the grafted tumor tissues was also examined using RT-qPCR. The RT-qPCR analysis revealed that the expression levels of miR-137 were significantly increased in the groups treated with the miR-137 mimics compared with the negative control treatment group $(\mathrm{P}<0.01 ;$ Fig. $5 \mathrm{D})$. These results suggested that the overexpression of miR-137 may inhibit tumor growth of EOC cells in vivo.

\section{Discussion}

In the present study, the results revealed that miR-137 was downregulated in ovarian cancer cell lines and tumor tissues, compared with normal OSE cells and non-tumorous ovarian tissues. Furthermore, the results also demonstrated that miR-137 inhibited EOC cell migration and invasion, which may be involved in the development of ovarian cancer metastasis. It was also demonstrated that miR-137 suppressed EOC tumor growth in nude mice. Therefore, it was hypothesized that low expression levels of miR-137 may be important in the progression of EOC.

It has been well-established that miR-137 is downregulated in various types of cancer (23-29). Several studies have reported that miR-137 may act as a tumor suppressor and inhibit cell proliferation, migration and invasion, and induce cell cycle arrest and apoptosis. Chen et al (32) demonstrated that miR-137 deregulation is common in glioma, and that restoration of its function inhibits cell proliferation and invasion by targeting cyclooxygenase (COX)-2. Liu et al (33) found that miR-137 suppresses cell growth and metastasis in hepatocellular carcinoma by directly targeting AKT2. Bi et al (34) found that the ectopic expression of miR-137 inhibits cell proliferation, induces cell apoptosis and suppresses cell migration and invasion in the A549 non-small cell lung cancer cell line by targeting paxillin. In the present study, it was observed that the expression level of miR-137 was low in ovarian cancer tissues and even lower in the metastatic ovarian tissues. In agreement with these results, Guo et al (23) demonstrated that miR-137 is downregulated in ovarian cancer tissues compared with normal ovarian samples, and that miR-137 negatively regulates the AEG-1 oncogene and suppresses ovarian cancer cell growth in vitro and in vivo. However, the role of miR-137 in ovarian cancer cell migration and invasion has not been investigated, to the best of our knowledge. Invasion and metastasis are two important attributes of malignant types of cancer, which are the cause of the high mortality rate in EOC (35). To the best of our knowledge, there are no previous reports regarding the role of miR-137 in migration and invasion in EOC. In this context, the present study revealed that the enforced expression of miR-137 inhibited the migration and invasion of ovarian cancer cells by inhibiting the expression of MMP-2 and MMP-9 expression, suggesting that miR-137 is important as a tumor suppressor in the motility of ovarian cancer cells.

It is important to note that one miRNA can exert different functions by targeting multiple mRNAs (36). To date, specific genes have been identified as gene targets of miR-137 in different tumors, including COX-2 in glioblastoma multiforme (28), cyclin-dependent kinase-6 in glioma cell lines (37) and lung cancer (38), Related to testes-specific, vespid and pathogenesis protein-1 in glioblastoma (39), C-terminal binding protein 1 in melanoma cells (40), estrogen-related receptor $\alpha$ in breast cancer $(29,41)$, cell division control protein 42 homolog in gastric cancer cells (32), colorectal cancer cells (42) and lung cancer (38) and c-Met, Y box binding protein 1, enhancer of zeste homolog 2 and microphthalmia-associated transcription factor in melanoma cells (43). Therefore, the present study hypothesized that miR-137 decreased cell proliferation, clonogenicity, migration and invasion, and induced G1 arrest and cell apoptosis via targeting multiple genes.

In conclusion, the findings of the present study demonstrated that miR-137 was downregulated in ovarian cancer cell lines and tumor tissues, compared with normal OSE cells and non-tumorous ovarian tissues, and that the overexpression of miR-137 inhibited cell proliferation, clonogenicity, migration, and invasion; induced G1 arrest and cell apoptosis in vitro and suppressed tumor growth in vivo. These findings suggested that miR-137 may be a potential therapeutic target for the treatment of EOC.

\section{Acknowledgements}

The present study was supported provided by The Science and Technology Research and Innovation team funded by Jilin province (grant no. JL2012048).

\section{References}

1. Jemal A, Siegel R, Ward E, et al: Cancer statistics, 2006. CA Cancer J Clin 56: 106-130, 2006.

2. Cannistra SA: Cancer of the ovary. N Engl J Med 351: 2519-2529, 2004.

3. Heintz AP, Odicino F, Maisonneuve P, et al: Carcinoma of the ovary. FIGO 26th annual report on the results of treatment in gynecological cancer. Int J Gynaecol Obstet 95 (Suppl 1): 161-192, 2006.

4. Wang M, He Y, Shi L and Shi C: Multivariate analysis by Cox proportional hazard model on prognosis of patient with epithelial ovarian cancer. Eur J Gynaecol Oncol 32: 171-177, 2011.

5. Djuranovic S, Nahvi A and Green R: A parsimonious model for gene regulation by miRNAs. Science 331: 550-553, 2011.

6. Kasinski AL and Slack FJ: Epigenetics and genetics. MicroRNAs en route to the clinic: progress in validating and targeting microRNAs for cancer therapy. Nat Rev Cancer 11: 849-864, 2011.

7. Iorio MV, Visone R, Di Leva G, et al: MicroRNA signatures in human ovarian cancer. Cancer Res 67: 8699-8707, 2007. 
8. Iorio MV and Croce CM: MicroRNAs in cancer: small molecules with a huge impact. J Clin Oncol 27: 5848-5856, 2009.

9. Bartel DP: MicroRNAs: genomics, biogenesis, mechanism and function. Cell 116: 281-297, 2004.

10. Lin J, Huang S, Wu S, et al: MicroRNA-423 promotes cell growth and regulates $\mathrm{G}(1) / \mathrm{S}$ transition by targeting $\mathrm{p} 21 \mathrm{Cip} 1 /$ Waf1 in hepatocellular carcinoma. Carcinogenesis 32: 1641-1647, 2011.

11. Jovanovic $M$ and Hengartner MO: miRNAs and apoptosis: RNAs to die for. Oncogene 25: 6176-6187, 2006.

12. Stevanato L and Sinden JD: The effects of microRNAs on human neural stem cell differentiation in two- and three-dimensional cultures. Stem Cell Res Ther 5: 49, 2014.

13. Ghosh G, Subramanian IV, Adhikari N, et al: Hypoxia-induced microRNA-424 expression in human endothelial cells regulates HIF-alpha isoforms and promotes angiogenesis. J Clin Invest 120 : 4141-4154, 2010

14. Schommer C, Palatnik JF, Aggarwal P, et al: Control of jasmonate biosynthesis and senescence by miR319 targets. PLoS Biol 6 : e230, 2008

15. Iorio MV and Croce CM: Causes and consequences of microRNA dysregulation. Cancer J 18: 215-222, 2012.

16. Esquela-Kerscher A and Slack FJ: Oncomirs-microRNAs with a role in cancer. Nat Rev Cancer 6: 259-269, 2006.

17. Tang W, Jiang Y, Mu X, Xu L, Cheng W and Wang X: MiR-135a functions as a tumor suppressor in epithelial ovarian cancer and regulates HOXA10 expression. Cell Signal 26: 1420-1426, 2014.

18. Zhang H, Wang Q, Zhao Q and Di W: MiR-124 inhibits the migration and invasion of ovarian cancer cells by targeting SphK1. J Ovarian Res 6: 84, 2013.

19. Bhattacharya R, Nicoloso M, Arvizo R, et al: MiR-15a and MiR-16 control Bmi-1 expression in ovarian cancer. Cancer Res 69: 9090-9095, 2009.

20. Hong F, Li Y, Xu Y and Zhu L: Prognostic significance of serum microRNA-221 expression in human epithelial ovarian cancer J Int Med Res 41: 64-71, 2013.

21. Corney DC, Hwang CI, Matoso A, et al: Frequent downregulation of miR-34 family in human ovarian cancers. Clin Cancer Res 16: 1119-1128, 2010.

22. Zhang W, Wang Q, Yu M, Wu N and Wang H: MicroRNA-145 function as a cell growth repressor by directly targeting c-Myc in human ovarian cancer. Technol Cancer Res Treat 13: 161-168, 2014

23. Guo J, Xia B, Meng F and Lou G: miR-137 suppresses cell growth in ovarian cancer by targeting AEG-1. Biochem Biophys Res Commun 441: 357-363, 2013.

24. LiP, Ma L, Zhang Y, Ji F and Jin F: MicroRNA-137 down-regulates KIT and inhibits small cell lung cancer cell proliferation. Biomed Pharmacother 68: 7-12, 2014.

25. Kozaki K, Imoto I, Mogi S, Omura K and Inazawa J: Exploration of tumor-suppressive microRNAs silenced by DNA hypermethylation in oral cancer. Cancer Res 68: 2094-2105, 2008

26. Ando T, Yoshida T, Enomoto S, et al: DNA methylation of microRNA genes in gastric mucosae of gastric cancer patients: its possible involvement in the formation of epigenetic field defect. Int J Cancer 124: 2367-2374, 2009.
27. Sun Y, Zhao X, Zhou Y and Hu Y: miR-124, miR-137 and miR-340 regulate colorectal cancer growth via inhibition of the Warburg effect. Oncol Rep 28: 1346-1352, 2012.

28. Chen L, Wang X, Wang $\mathrm{H}$, et al: miR-137 is frequently down-regulated in glioblastoma and is a negative regulator of Cox-2. Eur J Cancer 48: 3104-3111, 2012.

29. Zhao Y, Li Y, Lou G, et al: MiR-137 targets estrogen-related receptor alpha and impairs the proliferative and migratory capacity of breast cancer cells. PLoS One 7: e39102, 2012.

30. Mehta SP, Jose P, Mirza A, Pritchard SA, Hayden JD and Grabsch HI: Comparison of the prognostic value of the 6th and 7th editions of the Union for International Cancer Control TNM staging system in patients with lower esophageal cancer undergoing neoadjuvant chemotherapy followed by surgery. Dis Esophagus 26: 182-188, 2013.

31. Wu B, Li S, Sheng L, et al: Metformin inhibits the development and metastasis of ovarian cancer. Oncol Rep 28: 903-908, 2012.

32. Chen Q, Chen X, Zhang M, Fan Q, Luo S and Cao X: miR-137 is frequently down-regulated in gastric cancer and is a negative regulator of Cdc42. Dig Dis Sci 56: 2009-2016, 2011.

33. Liu LL, Lu SX, Li M, et al: FoxD3-regulated microRNA-137 suppresses tumour growth and metastasis in human hepatocellular carcinoma by targeting AKT2. Oncotarget 5: 5113-5124, 2014.

34. Bi Y, Han Y, Bi H, Gao F and Wang X: miR-137 impairs the proliferative and migratory capacity of human non-small cell lung cancer cells by targeting paxillin. Hum Cell 27: 95-102, 2014.

35. Hainaut $\mathrm{P}$ and Plymoth A: Targeting the hallmarks of cancer: towards a rational approach to next-generation cancer therapy. Curr Opin Oncol 25: 50-51, 2013.

36. Garzon R, Marcucci G and Croce CM: Targeting microRNAs in cancer: rationale, strategies and challenges. Nat Rev Drug Discov 9: 775-789, 2010.

37. Silber J, Lim DA, Petritsch C, et al: miR-124 and miR-137 inhibit proliferation of glioblastoma multiforme cells and induce differentiation of brain tumor stem cells. BMC Med 6: 14, 2008.

38. Zhu X, Li Y, Shen H, et al: miR-137 inhibits the proliferation of lung cancer cells by targeting Cdc42 and Cdk6. FEBS Lett 587: 73-81, 2013

39. Bier A, Giladi N, Kronfeld N, et al: MicroRNA-137 is downregulated in glioblastoma and inhibits the stemness of glioma stem cells by targeting RTVP-1. Oncotarget 4: 665-676, 2013.

40. Deng Y, Deng H, Bi F, et al: MicroRNA-137 targets carboxyl-terminal binding protein 1 in melanoma cell lines. Int J Biol Sci 7: 133-137, 2011.

41. Zhao Y, Li Y, Lou G, et al: MiR-137 targets estrogen-related receptor alpha and impairs the proliferative and migratory capacity of breast cancer cells. PLoS One 7: e39102, 2012.

42. Liu M, Lang N, Qiu M, et al: miR-137 targets Cdc42 expression, induces cell cycle G1 arrest and inhibits invasion in colorectal cancer cells. Int J Cancer 128: 1269-1279, 2011.

43. Luo C, Tetteh PW, Merz PR, et al: miR-137 inhibits the invasion of melanoma cells through downregulation of multiple oncogenic target genes. J Invest Dermatol 133: 768-775, 2013. 